\title{
DIFFUSE INTERSTELLAR FEATURES IN THE SPECTRA OF DUST-EMBEDDED STARS
}

\author{
G. E. BROMAGE* and K. NANDY \\ Royal Observatory, Edinburgh, U.K.
}

\begin{abstract}
Eleven diffuse interstellar features have been studied in the spectra of dust-embedded stars in Cyg OB2 (VI Cyg) (including a Wolf-Rayet star) and field stars in the same direction. The equivalent widths of the lines are all strongly correlated with each other and with $E_{B-V}$, but there is virtually no correlation with $\mathrm{D}$ line strength for these stars. The wavelength positions and half-widths of the diffuse lines appear to be the same for all stars studied.
\end{abstract}

A programme of extending observations to stars as faint as $B=14 .^{m} 0$ for the study of diffuse interstellar features is being continued. The object of this programme is to determine whether any systematic differences exist between the relative strengths and positions of the features produced in circumstellar clouds, and those produced in the general interstellar medium.

Observations of eleven diffuse features in the wavelength range $5500-7000 \AA$ are presented here for dust-embedded stars in the association Cyg OB2 (VI Cyg) and for field stars in the same direction.

TABLE I

Stars observed

\begin{tabular}{|c|c|c|c|c|}
\hline Star & Assn. & Sp. Type & $V$ & $E_{B-V}$ \\
\hline HDE 229059 & Cyg OB1 & B1.5Ia & 8.70 & 1.71 \\
\hline HD 194279 & Cyg OB9 & B1.5Ia & 7.02 & 1.20 \\
\hline HD 194839 & & B0.5Ia & 7.49 & 1.19 \\
\hline HD 195229 & & B0.5III & 7.66 & 0.44 \\
\hline HD 195592 & & O9.5Ia & 7.08 & 1.14 \\
\hline $\mathrm{BD}+40^{\circ} 4219$ & Cyg OB2 4 & 08 & 10.22 & 1.50 \\
\hline $\mathrm{BD}+40^{\circ} 4220$ & Cyg OB2 5 & O7f & $9.2 \mathrm{var}$ & 2.02 \\
\hline $\mathrm{BD}+40^{\circ} 4227$ & Cyg OB2 8A & O6f & 9.03 & 1.59 \\
\hline \multirow[t]{3}{*}{$\mathrm{BD}+41^{\circ} 3804$} & Cyg OB2 10 & 09.5Ia & 9.88 & 1.80 \\
\hline & Cyg OB2 12 & B5Ia-O & 11.51 & 3.44 \\
\hline & Cyg OB2 WR2 & WN5 & 11.8: & 2.4: \\
\hline HD 199478 & & B8Ia & 5.68 & 0.47 \\
\hline HD 202850 & Cyg OB4 & B9Iab & 4.23 & 0.11 \\
\hline
\end{tabular}

The spectra were obtained at a dispersion of $60 \AA \mathrm{mm}^{-1}$ and a resolution of $2 \AA$ with the Cassegrain spectrograph of the $98^{\prime \prime}$ Isaac Newton telescope. The Cygnus stars observed are listed in Table I.

* Now at Department of Structural Chemistry, University of Bradford, Bradford, 7, Yorkshire, England. 
The spectra are measured in terms of Baker density

$$
\Delta=\log _{10}\left(\frac{1}{T}-1\right)
$$

and a calibration curve is derived for the wavelength of each feature. The profile of the broad feature at $6176 \AA$ is obtained by the comparison of stars of similar spectral type. Equivalent widths are derived directly from the digitised spectra: spectral densities are reduced to intensities and then rectified by linearly interpolating the continuum over the integration region for each feature. The same integration regions are used for all stars: they are listed in Table II. The integration steplength is $\frac{1}{2} \AA$.

For the narrow features, the error on an equivalent width estimate arises mainly from the emulsion granularity; but for the two broad ones, the uncertainty in the continuum position is more important. Errors from other sources (such as calibration uncertainties and corrections for blends with stellar and telluric lines) rarely amount to more than $3 \%$ of the total equivalent width. Standard errors for all stars have been computed from the data for those with 3 or more spectra.

The features appear very strong in the spectra of the heavily reddened stars of Cyg OB2, which range in spectral type from Wolf-Rayet and Of to B5Ia. A microdensitometer trace of the spectrum of the Wolf-Rayet star No. WR2 (Herbig and Mendoza, 1960), which is believed to be a member of the association, is shown in Figure 1a. From the slope of the continuum, the $(B-V)$ colour is estimated to be $2.1 \pm 0.2$, which agrees with the value obtained by Reddish (1968) from UBV photometry. This star was previously classified as WN7, but from the observed ratio of C IV to $\mathrm{He}$ I emission line strengths, it must be WN5.

The 07f star Cyg OB2 No. 5, which is an eclipsing binary, shows $\mathrm{H} \alpha$ and He I ( $\lambda$ 6678) in emission and He I ( $\lambda$ 5875) in absorption, all shifted to the blue by approx-

TABLE II

\begin{tabular}{ll}
\multicolumn{2}{c}{ Spectrum lines measured } \\
\hline Line name & $\begin{array}{l}\text { Integration region } \\
(\mathrm{nm})\end{array}$ \\
\hline $5705(+\mathrm{N}$ II 571.1$)$ & $570.1-571.5$ \\
N II 571.1 only & $570.8-571.5$ \\
$5780+5776$ & $575.5-579.1$ \\
5780 only & $577.3-578.7$ \\
$5797(+\mathrm{C}$ IV 580.2) & $579.1-580.5$ \\
C IV 581.2 & $580.8-581.5$ \\
D lines (Na I) & $588.3-590.3$ \\
6010 & $600.4-601.6$ \\
$6176+6203$ & $613.5-622.0$ \\
6203 only & $619.7-620.9$ \\
6270 & $626.5-627.4$ \\
$6284\left(+\mathrm{O}_{2} 627.8\right)$ & $627.4-629.5$ \\
6379 & $637.5-638.3$ \\
6614 & $660.8-662.0$ \\
\hline
\end{tabular}




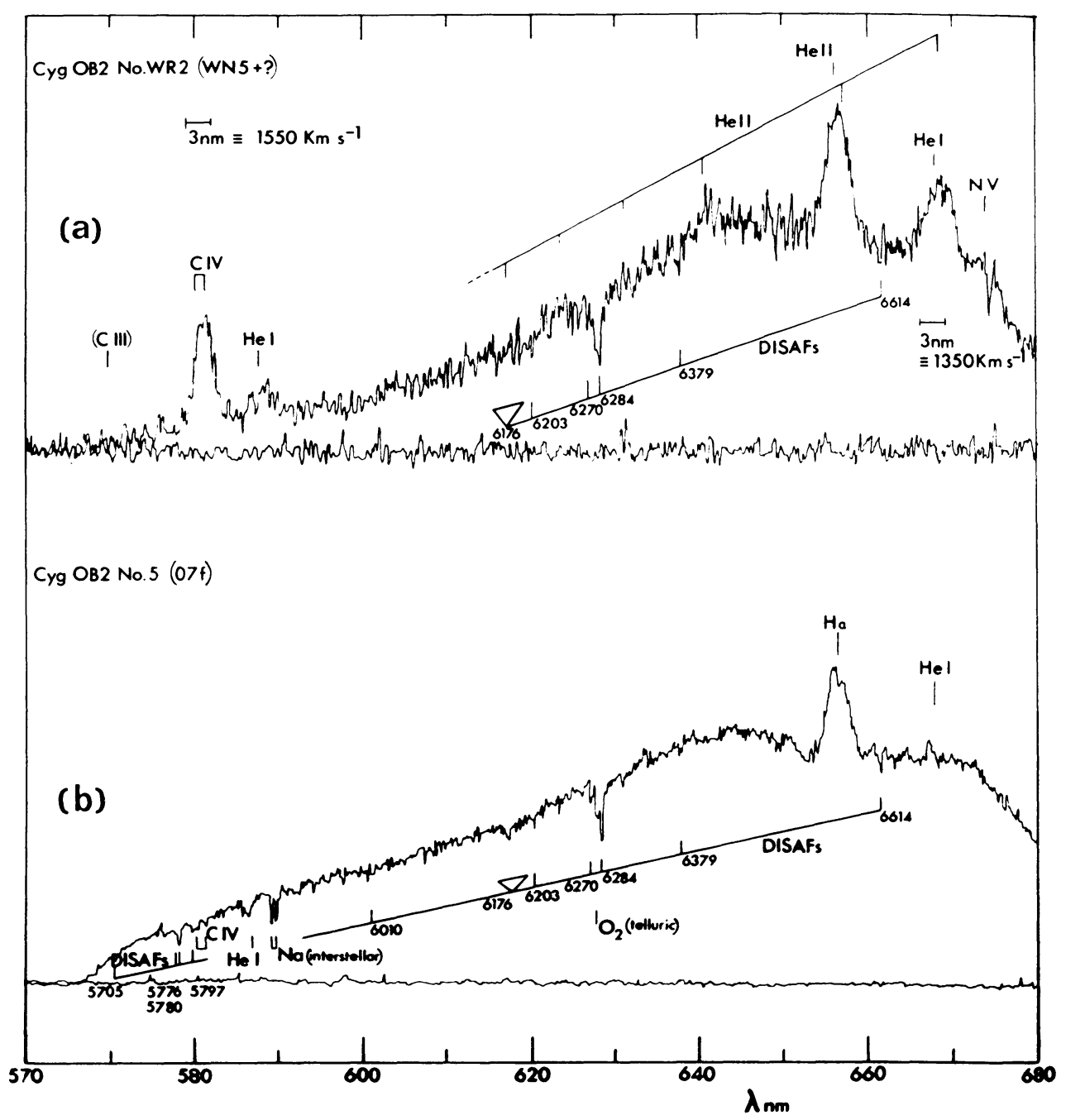

Fig. 1. Microdensitometer tracings of the spectra of two members of Cyg OB2: (a) No. WR2, type WN5, $V=11.8, E_{B-V}=2.4, M_{V} \simeq-6$. (b) No. 5, type $07 f, V=9.2, E_{B-V}=2.0, M_{V} \simeq-8$.

imately $150 \mathrm{~km} \mathrm{~s}^{-1}$ (see Figure $1 \mathrm{~b}$ ). It is interesting to note that the $\mathrm{C}$ IV absorption lines ( $5802 \AA$ and $5712 \AA$ ), on the contrary, do not seem to show the same shift.

The most heavily reddened star Cyg OB2 No. 12 shows the strongest diffuse features in the red so far recorded (Bromage, 1971), and also has $\mathrm{H} \alpha$ in emission. A microdensitometer tracing of the spectrum of this star is reproduced in Figure 2. The ' 6284 ' line has a central depth of almost $50 \%$.

A result which is of considerable interest is that for all the stars studied here, whether dust embedded or field stars, the wavelength positions of the observed diffuse features are the same, within the spectral resolution. 


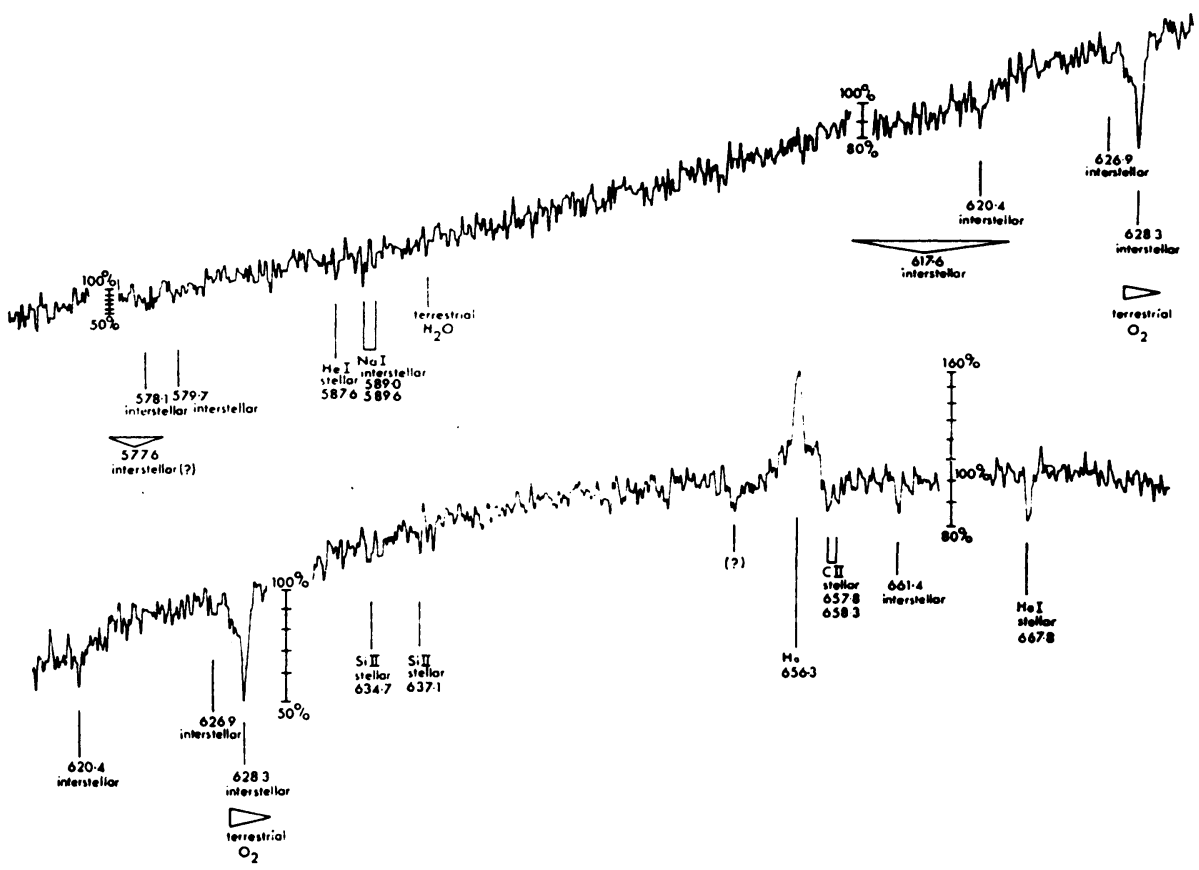

Fig. 2. Microdensitometer tracing of the spectrum of the star Cyg OB2 No. 12, type B5 Ia-O, $V=11.5, E_{B-V}=3.5, M_{V} \simeq-12$. Upper trace, wavelength range $\lambda \lambda 575-630 \mathrm{~nm}$; lower trace, $\lambda \lambda 620-675 \mathrm{~nm}$.
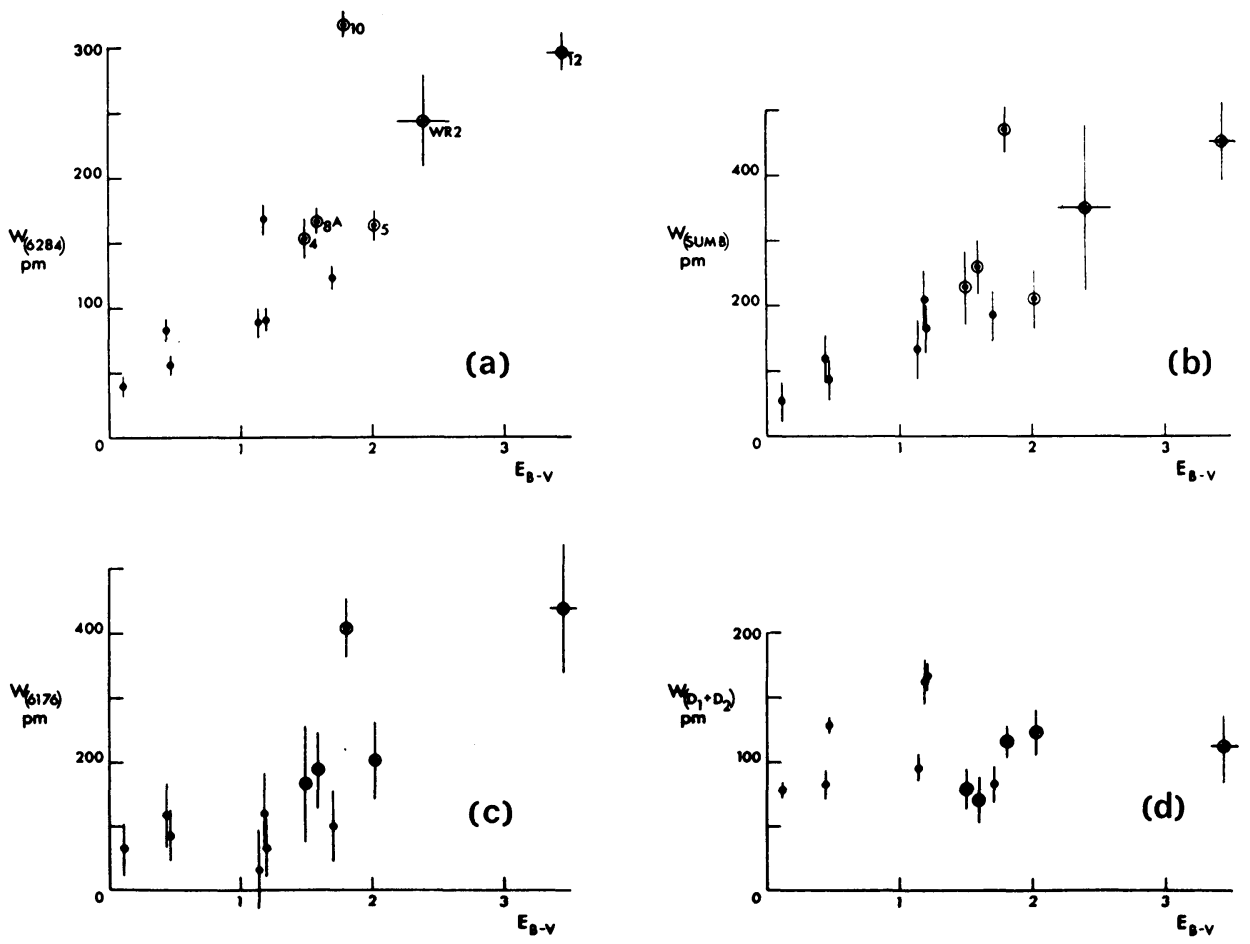

Fig. 3. Graphs of equivalent widths of interstellar lines (in picometres $\equiv 0.01 \AA$ ) against $E_{B-\nu}$, for Cygnus stars. Points referring to members of Cyg OB2 are shown as open circles. The error bars refer to standard errors. (a) The typical narrow diffuse line '6284' (half-width $\simeq 0.4 \mathrm{~nm}$ ); (b) The total equivalent width of 5 narrow lines in the range $\lambda \lambda$ 620-665 (SUM B); (c) The typical broad diffuse feature ' 6176 ' (half-width $\simeq 2.5 \mathrm{~nm}$ ); (d) The total equivalent width of the interstellar sodium D lines. https://doi.org/10.1017/S007418090005405X Published online by Cambridge University Press 

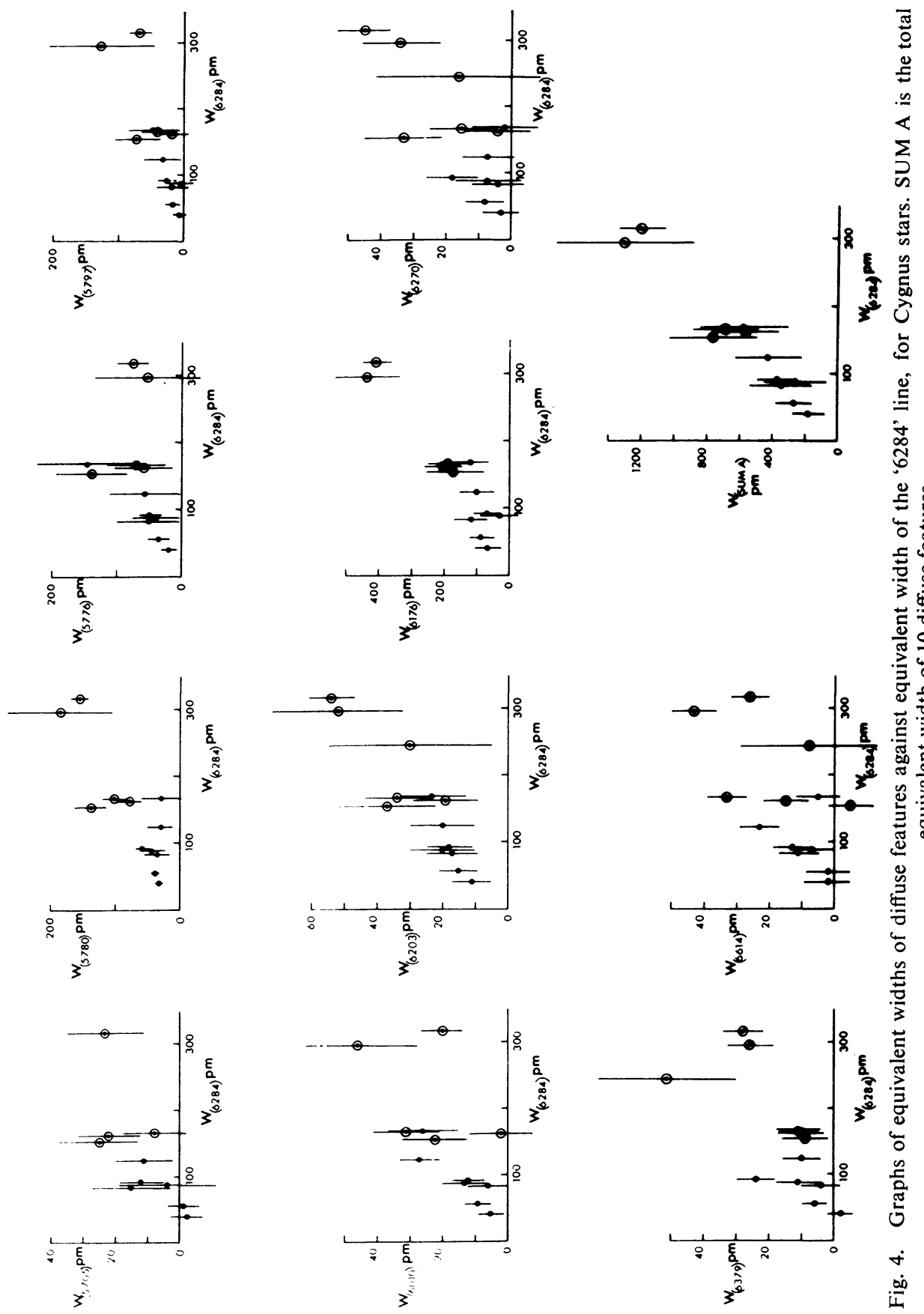
The relations between the equivalent widths of the typical narrow line '6284' and the broad one ' 6176 ', and colour excess $E_{B-V}$, are shown in Figure 3. In general, they appear to be linearly related over the whole range of colour excess, with the exception of Cyg OB2 No. 10. The total equivalent width of 5 narrow lines (denoted by Sum B in Figure 3b) also increases linearly with $E_{B-V}$. Notice in particular that the WolfRayet star and No. 12 both have normal line strengths.

While the strengths of the diffuse features systematically increase with colour excess, the interstellar sodium $D$ lines have approximately the same equivalent width over the whole range of $E_{B-V}$ observed, as shown in Figure 3. This implies, since the $\mathrm{D}$ lines are too weak to be saturated, that the reddening of the association members is due to a localised dust cloud or clouds which are deficient in sodium gas. The sodium lines seem to be produced only in the general interstellar medium.

To study the inter-correlation of these features, the equivalent width of each line, and the total equivalent width of 10 diffuse features (both narrow and broad), are plotted in Figure 4 against that of the strongest and most accurately measured narrow feature ' 6284 '. The overall picture is that they are all strongly correlated. In addition a strong correlation of the feature '6284' with the broad diffuse band ' 4430 ' has been found by Murdin (1972).

The profile of the broad diffuse band at $\lambda 6176$ has been obtained from the comparison of the spectra of Cyg OB2 No. 10 and $\kappa$ Cas, and is shown in Figure 5. The apparent emission wing at the blue side of the band is similar to that seen in ' 4430 ' (Brück and Nandy, 1968; Bromage, 1972).

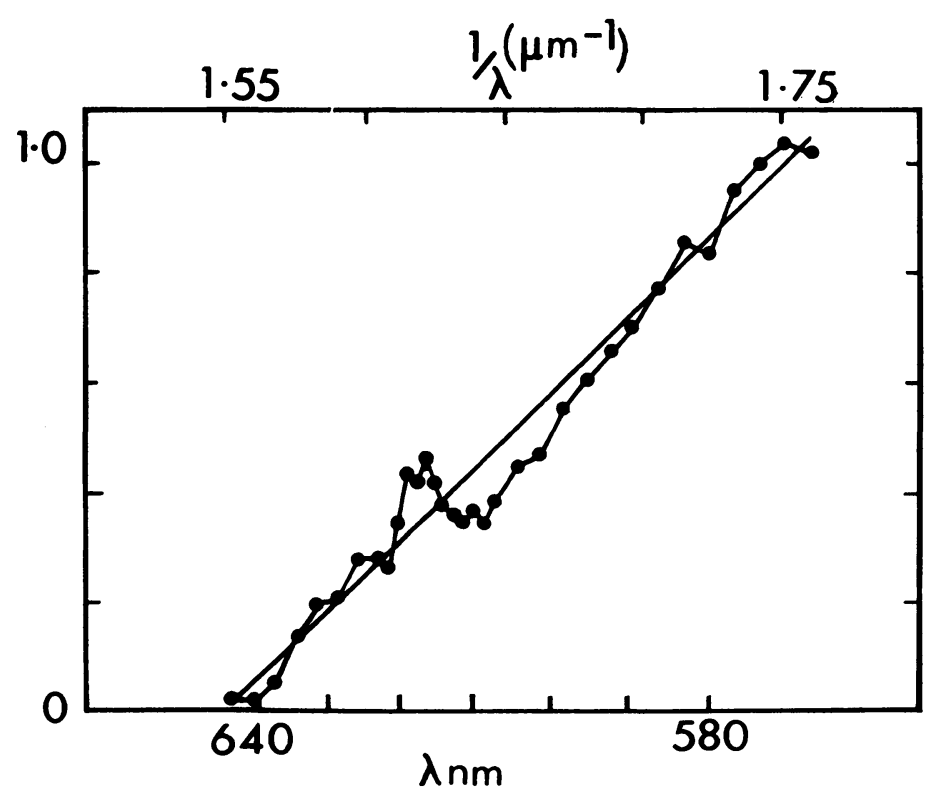

Fig. 5. The profile of the broad ' 6176 ' feature for Cyg OB2 No. 10 compared with $\varkappa$ Cas $\left(\triangle E_{B-V}=\right.$ 1.47). 
Our interpretation of these observations is that the strong correlation of strength of diffuse features with colour excess, the profiles of the broad features, and the intercorrelation of the strength of the features, together with no correlation with $\mathrm{D}$ line strength, all support the view that these features are produced by the grains rather than the gas. However, the invariance of the wavelength positions and of the halfwidths of these features requires a high degree of uniformity of physical conditions of the grains, including temperature, effective size and nature of the impurities.

\section{References}

Bromage, G. E.: 1971, Nature 230, 172.

Bromage, G. E.: 1972, Astrophys. Space Sci. 15, 426.

Brück, M. T. and Nandy, K.: 1968, Nature 220, 46.

Herbig, G. M. and Mendoza, E. E.: 1960, Bol. Obs. Tonatzintla y Tacubaya, No. 19, p. 21.

Murdin, P.: 1972, Monthly Notices Roy. Astron. Soc. 157, 461.

Reddish, V. C.: 1968, Observatory 88, 139. 